\title{
MIÍASES HUMANAS POR Cochliomyia hominivorax (COQUEREL, 1858) (DIPTERA, CALLIPHORIDAE) EM HOSPITAIS PÚBLICOS NA CIDADE DO RECIFE, PERNAMBUCO, BRASIL
}

Edleuza Maria Ferreira do Nascimento(1), Jaqueline Bianque de Oliveira(1), Maria José Paes(1), Andrea de Paula Lobo(1), Ana Lúcia Andrade da Silva(1), Edivaldo Rosas dos Santos Júnior ${ }^{(1)}$, Jefferson Luis Figueiredo Leal ${ }^{(1)} \&$ Gonzalo Efraim Moya-Borja ${ }^{(2)}$

\section{Abstract}

Human myiasis by Cochliomyia hominivorax (Coquerel, 1858) (Diptera, Calliphoridae) in Recife, State of Pernambuco, Brazil - The occurence of human myiasis caused by larvae of Cochliomyia hominivorax was registered for the first time in Recife, State of Pernambuco, Brazil. From November, 1999 to October, 2002 were registered, in three public hospitals 24 cases of myiasis in human beings, of both sexes, with age varying from 08 to 93 years old. The wounds infested with screwworms observed in these patients were classified as cutaneous, oral, anal, aureal and ocular myiasis. The inferior members were more frequently attacked $(10 / 24)(41.7 \%)$ than the other anatomic areas. In all of the cases, the

(1) Laboratório de Parasitologia, Departamento de Biologia, Universidade Federal Rural de Pernambuco, R. Dom Manoel de Medeiros s/n, CEP: 52171-030 Recife, Pernambuco, Brasil. E-mail: jackie@ufrpe.br; (2) Laboratório de Entomologia das Miíases Neotropicais, Instituto de Biologia, Universidade Federal Rural do Rio de Janeiro, Km 47 da antiga rodovia Rio-São Paulo, CEP: 2389-000, Seropédica, Rio de Janeiro, Brasil.

Recebido em: 12/07/2004

Aceito em: 16/03/2005. 
myiasis were established in pre-existent lesions, and the factors that predispose to this disease were: excoriations of varied nature (10/24) (41.7\%); no identified lesions (05/24) $(20.8 \%)$; diseases of the circulatory system (03/24) (12.5\%); tumors (02/24) (8.3\%); conjunctivitis, auricular infections, dermatitis and surgical wound $(01 / 24)(4.2 \%)$. The factors that predispose to be infested by $C$. hominivorax were the physical and mental weakness, dehydration, inadequate corporal hygiene, diabetes, malnutrition, elephantiasis, schizophrenia, alcoholism, anemia, lice infestation and fundamentally accidental wounds.

Key words: Human myiasis, C. hominivorax, Calliphoridae, Pernambuco, Brazil

\section{Resumo}

A ocorrência de miíases humanas causadas pelas larvas de Cochliomyia hominivorax foi registrada pela primeira vez em Recife, Estado de Pernambuco, Brasil. No período de novembro de 1999 a outubro de 2002 foram registrados, em três hospitais da rede pública, 24 casos de miíases em pacientes de ambos os sexos, com idade variando de 08 a 93 anos. As miíases diagnosticadas foram classificadas em cutâneas, orais, anais, auriculares e oculares, sendo os membros inferiores acometidos com maior freqüência (10/24) (41,7\%). Em todos os casos, as miíases se desenvolveram em lesões pré-existentes, resultantes de: escoriações de natureza variada (10/24) (41,7\%); lesões de origem não identificada (05/ 24) $(20,8 \%)$; doenças do sistema circulatório (03/24) (12,5\%); neoplasias (02/24) (8,3\%); conjuntivite, otite, dermatite e ferida cirúrgica (01/24) (4,2\%). Os fatores predisponentes às míiases foram debilidade física e mental, desidratação, higiene corporal inadequada, diabetes, desnutrição, elefantíase, esquizofrenia, alcoolismo, anemia, infestação por piolhos e, fundamentalmente, feridas acidentais.

Palavras-chave: Miíase humana, Cochliomyia hominivorax, Calliphoridae, Pernambuco 


\section{Introdução}

O termo mí́ase foi proposto por Hope em 1840 para descrever a invasão de tecidos ou órgãos do homem ou de animais por larvas de dípteros (Guimarães \& Papavero, 1999). Hall \& Wall (1995) realizaram uma revisão de literatura sobre a classificação das miíases, bem como estudos sobre a evolução, distribuição, fisiologia e técnicas de controle das miíases dos humanos e dos animais domésticos no mundo. As larvas de Cochliomyia hominivorax (Coquerel, 1858) e Dermatobia hominis (Wiedemann, 1819) são os agentes causais mais comuns das miíases humanas na América (Guimarães \& Papavero, 1999). Estas duas espécies são originárias da América e constituem os parasitos mais importantes dos animais domésticos e selvagens (Moya-Borja, 2003). Em 1935 nos EUA foram relatados 230.000 casos de miíases em animais e unicamente 100 casos em humanos (James, 1947). A C. hominivorax tem sido erradicada dos EUA e da maioria dos países da América Central usando a técnica de liberação dos machos sexualmente estéreis (Graham, 1985). Esta espécie foi introduzida acidentalmente no Norte da África (Líbia) e poucos casos humanos foram registrados no período de 1988 a 1990; para evitar desastres imprevisíveis nos animais domésticos e selvagens e com a população humana, mais de 600 milhões de moscas estéreis foram liberadas no Norte da África, atingindo-se a erradicação de $C$. hominivorax em junho de 1991 (Cunningham, 1991).

$\mathrm{Na}$ América do Sul ainda são registrados casos de míiases provocadas por $C$. hominivorax. Em geral, os pacientes acometidos apresentam fatores predisponentes tais como higiene pessoal deficiente, respiração bucal durante o sono, desnutrição, retardo mental, alcoolismo, senilidade, hemiplegia e traumatismos (Durighetto Jr. et al., 1995; Stephan \& Fuentefria, 1999; García et al., 2002). Diante da escassez de informações acerca das miíases humanas no Brasil e da inexistência destes dados na região Nordeste, este trabalhou objetivou identificar as espécies e os fatores envolvidos na ocorrência de mí́ases em pacientes atendidos em hospitais da rede pública na cidade do Recife, como parte do projeto de identificação de dípteros ciclorrafos do Estado de Pernambuco. 


\section{Material e Métodos}

Este estudo foi realizado no período de novembro de 1999 a outubro de 2002 em três hospitais localizados na cidade do Recife, pertencentes à rede pública do estado de Pernambuco: Hospital Getúlio Vargas (HGV), Hospital Casa de Misericórdia (HCM) e Hospital da Restauração (HR). Os pacientes, de ambos os sexos e com idade variando de 08 a 93 anos, após o exame clínico e diagnóstico, foram conduzidos para a sala de expurgo onde as larvas eram retiradas das lesões com auxílio de pinça e conservadas em recipientes identificados contendo álcool 70으. Em alguns casos, para coleta das larvas que se encontravam profundamente inseridas nos tecidos, utilizou-se gaze embebida em éter que era colocada em torno ou sobre a lesão.

Para identificação das larvas, foram consideradas as características morfológicas das placas estigmáticas e das aberturas espiraculares (García et al., 2002). Algumas larvas após coletadas, foram colocadas em placas de Petri contendo areia, no interior de uma caixa de madeira $(20 \mathrm{x}$ $20 \mathrm{~cm}$ ) revestida com tela de nylon, e ali permaneciam até a emergência das moscas adultas. Os dípteros, adultos e larvas, foram identificados no Laboratório de Parasitologia do Departamento de Biologia da Universidade Federal Rural de Pernambuco (UFRPE).

Os pacientes e/ou seus parentes, foram entrevistados para obtenção de informações sobre situação sócio-econômica (profissão, renda, moradia) e histórico clínico da lesão (como e quando iniciou). Mediante autorização dos pacientes, as lesões foram fotografadas, respeitando assim as normas de ética quando seres humanos estão envolvidos em pesquisas em beneficio de sua saúde e bem-estar.

\section{Resultados e Discussão}

Durante 35 meses de estudo, foram realizados 24 diagnósticos de míases, dos quais, 19 pacientes foram atendidos no HGV, 04 no HCM, e 01 no HR (Tab. I). Estudos sobre a ocorrência das miíases humanas são escassos, merecendo destaque o estudo epidemiológico pioneiro realizado em hospitais de Brisbane, onde foram registrados 14 casos em 36 meses de estudo (Lukin, 1989). Posteriormente, Kumarasinghe et al. (2000) relataram a ocorrência de 16 casos em 18 meses de estudos em hospitais 


\section{Tabela I}

Casos de miíases diagnosticados em pacientes humanos, de ambos os sexos, atendidos em três hospitais da rede pública na cidade do RecifePE, no período de novembro de 1999 a outubro de 2002.

\begin{tabular}{cccc}
\hline Hospital & Masculino & Feminino & Total \\
\hline HGV & 12 & 07 & 19 \\
HCM & 01 & 03 & 04 \\
HR & 01 & - & 01 \\
Total & 14 & 10 & 24 \\
\hline $\begin{array}{l}\text { HGV - Hospital Getúlio Vargas; HCM - Hospital Casa } \\
\text { de Misericórdia; HR - Hospital da Restauração. }\end{array}$
\end{tabular}

no Sri Lanka, enquanto, em hospitais nos Estados Unidos, Sherman (2000) registrou a ocorrência de 42 casos no período de 36 meses.

Em todos os casos diagnosticados, as larvas e os adultos foram identificados como sendo Cochliomyia hominivorax (Diptera, Calliphoridae). De acordo com sua biologia, as larvas de $C$. hominivorax são classificadas como biontófagas ou obrigatórias, pois são capazes de invadir e se desenvolver em feridas recentes ou em aberturas naturais, determinando rápida e intensa destruição tecidual (Schreiber et al., 1964; Durighetto Jr. et al., 1995; Nogueira et al., 2000; Sherman, 2000; García et al., 2002). A sintomatologia e a gravidade, além das seqüelas, variam de acordo com a localização, número de larvas e estado geral dos pacientes (Durighetto Jr. et al., 1995; Nogueira et al., 2000). O envolvimento de $C$. hominivorax era esperado uma vez que as infestações se instalaram em lesões ulcerativas do tecido cutâneo e de mucosas e as larvas coletadas encontravam-se profundamente inseridas em tecidos vivos, não sendo encontradas em tecido necrosado. Em aproximadamente $44 \%$ dos casos de miíases humanos diagnosticados nos Estados Unidos havia o envolvimento de $C$. hominivorax (Schreiber et al., 1964; Sherman, 2000). No entanto, desde 1973 não são relatados, nos Estados Unidos, casos de miíases humanas por esta espécie como conseqüência do programa de erradicação adota- 
do (Sherman, 2000). Na América Latina, casos de miíases humanas por C. hominivorax e D. hominis foram relatados na Costa Rica, Chile e Argentina (Calderón et al., 1996; Apt et al., 2001; García et al., 2002). No Brasil, larvas de $C$. hominivorax foram identificadas nos casos de miíases orais relatados por Durighetto Jr. et al. (1995), Stephan \& Fuentefria (1999) e Nogueira et al. (2000). As miíases são mais freqüentes em áreas rurais uma vez que nestas áreas há uma maior proximidades entre o homem e as criações de animais (Sherman, 2000). Apesar da alta prevalência das miíases por $C$. hominivorax em áreas rurais, esta espécie é também abundante no perímetro urbano devido não só à presença de cães errantes que apresentam feridas freqüentes, resultantes de brigas pela disputa de fêmeas no cio, mas também da criação de outros animais que também apresentam ferimentos infestados. No entanto, no estudo de dinâmica populacional realizado por Lobo et al. (2001) na região metropolitana do Recife, os dípteros Calliphoridae foram os mais abundantes, apesar de espécimes adultos de $C$. hominivorax não terem sido capturados, o que também foi observado por Maciel et al. (2001) em Mossoró, Rio Grande do Norte. Segundo os referidos autores, este resultado pode ter sofrido interferência do tipo de armadilha e iscas utilizadas.

As miíases diagnosticadas foram classificadas em cutâneas, orais, anais, auriculares e oculares (Figs. 1-4), segundo os critérios de Sherman (2000) (Tab. II). Em relação aos sítios anatômicos, as míases ocorreram com maior freqüência nos membros inferiores $(41,7 \%)$, o que também foi observado por Kumarasinghe et al. (2000) e Sherman (2000) nos estudos realizados no Sri Lanka e Estados Unidos, respectivamente. As miíases ocorreram com menor freqüência na cabeça (16,7\%); cavidade oral (12,5\%); ânus, ouvido e olho (8,3\%), e, região dorsal $(4,2 \%)$ (Tab. III). Devido ao grau de infestação e comprometimento da área afetada, dos 04 dos 10 pacientes com miíases em membros inferiores tiveram como sequela a amputação de membros, e 01 dos 02 pacientes com miíase ocular teve perda da visão. Na Austrália, dos 14 pacientes analisados por Lukin (1989), 07 precisaram submeter-se à amputação. Neste sentido, destaca-se a importância do diagnóstico rápido e específico em função da agressividade das larvas biontófagas de C. hominivorax (García et al., 2002).

Vários são os fatores que predispõem a ocorrência das miíases humanas. Em todos os casos analisados neste estudo, as miíases se instalaram em feridas pré-existentes ou em aberturas naturais com exsudação e/ou odor fétido, e os fatores predisponentes observados fo- 
Tabela II

Classificação das miíases diagnosticadas em pacientes humanos atendidos em três hospitais da rede pública na cidade do Recife-PE, no período de novembro de 1999 a outubro de 2002.

\begin{tabular}{ccc}
\hline Classificação & Número de Casos & $\%$ \\
\hline Cutânea & 15 & $62,5 \%$ \\
Oral & 03 & $12,5 \%$ \\
Anal & 02 & $8,3 \%$ \\
Auricular & 02 & $8,3 \%$ \\
Ocular & 02 & $8,3 \%$ \\
Total & 24 & $100 \%$ \\
\hline
\end{tabular}

ram: escoriações de natureza variada (10/24) (41,7\%); lesões de origem não identificada (05/24) $(20,8 \%)$; doenças do sistema circulatório (03/24) $(12,5 \%)$; neoplasias $(02 / 24)(8,3 \%)$; conjuntivite, otite, abscesso, dermatite e ferida cirúrgica (01/24) $(4,2 \%)$. Na Tabela IV são apresentadas as principais informações clínicas dos 24 pacientes analisados. Nos estudos realizados por Kumarasinghe et al. (2000) e Sherman (2000), os fatores predisponentes mais comuns foram as dermatites e as doenças do sistema circulatório (doença vascular periférica, acidente vascular cerebral, etc...), respectivamente, enquanto Lukin (1989) destacou diabetes e as doenças vasculares. Outros fatores como debilidade física e/ou mental, diabetes, elefantíase, esquizofrenia, alcoolismo e infestação por piolhos, também estiveram associados com as miíases nos pacientes ora analisados. A literatura mundial relata que os pacientes acometidos por miíases, em geral apresentam alguns fatores predisponentes e associados tais como: higiene corporal e oral deficientes, lesões com exsudação e/ou odor fétidos, respiração bucal durante o sono, desnutrição, doenças psicológicas e retardamento mental, alcoolismo, senilidade, hemiplegia, hanseníase, leishmaniose, neoplasias, doenças vasculares, entre outros (Durighetto 


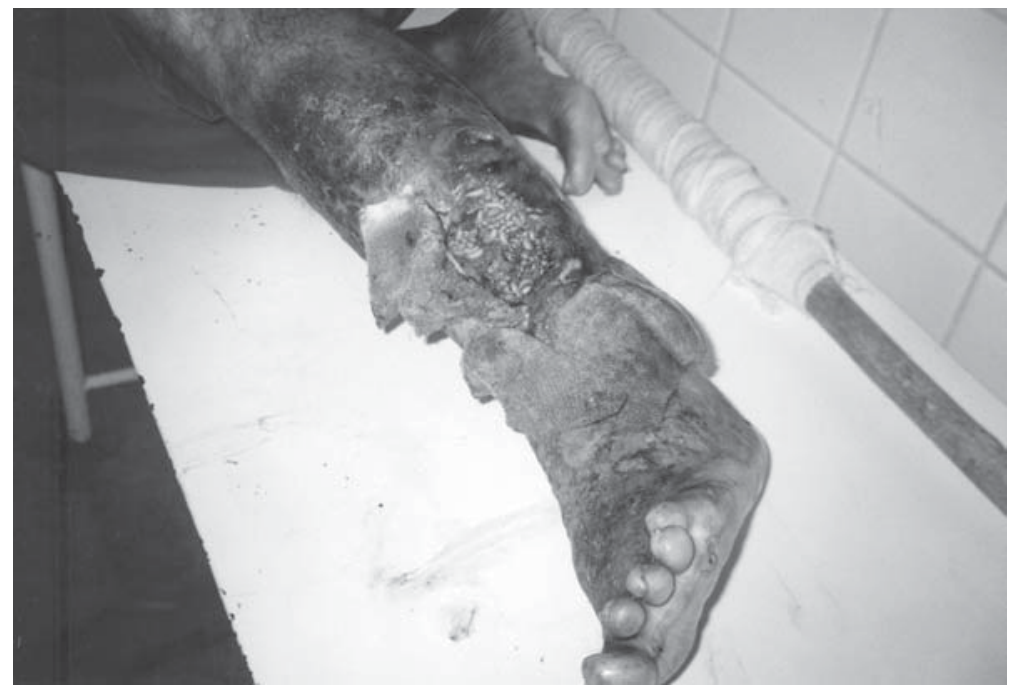

Figura 1: Miíase cutânea em paciente com ulceração vascular.

Jr. et al., 1995; Stephan \& Fuentefria, 1999; Kumarasinghe et al., 2000; Nogueira et al., 2000; Sherman, 2000). Lukin (1989) chamou a atenção para o fato de que seus pacientes eram idosos, doentes e debilitados. A ocorrência de um ou mais destes fatores, associado ao número de larvas e estado geral dos pacientes pode resultar em mutilações ou morte (Durighetto Jr. et al., 1995; Stephan \& Fuentefria, 1999; Nogueira et al., 2000). Além dos fatores anteriormente expostos, a situação sócio-econômica dos indivíduos, aliada ao desconhecimento da doença e a dificuldade de atendimento médico em hospitais públicos e postos de saúde, são potencializadores destas infestações e de suas conseqüências (Lukin, 1989; Stephan \& Fuentefria, 1999). Kumarasinghe et al. (2000) alegaram que as dermatites na região dos pés são freqüentes devido a não utilização de sapatos, o que se deve às precárias condições sócio-econômicas de 14 dos 16 pacientes com diagnóstico de miíase no Sri Lanka. Por outro lado, Sherman (2000) registrou que $38,1 \%$ dos seus pacientes eram moradores de rua e portadores de diabetes ou doença vascular. A maioria dos pacientes, analisados neste estudo, era constituída por trabalhadores assalariados ou aposentados, com moradia fixa, com exceção de um 


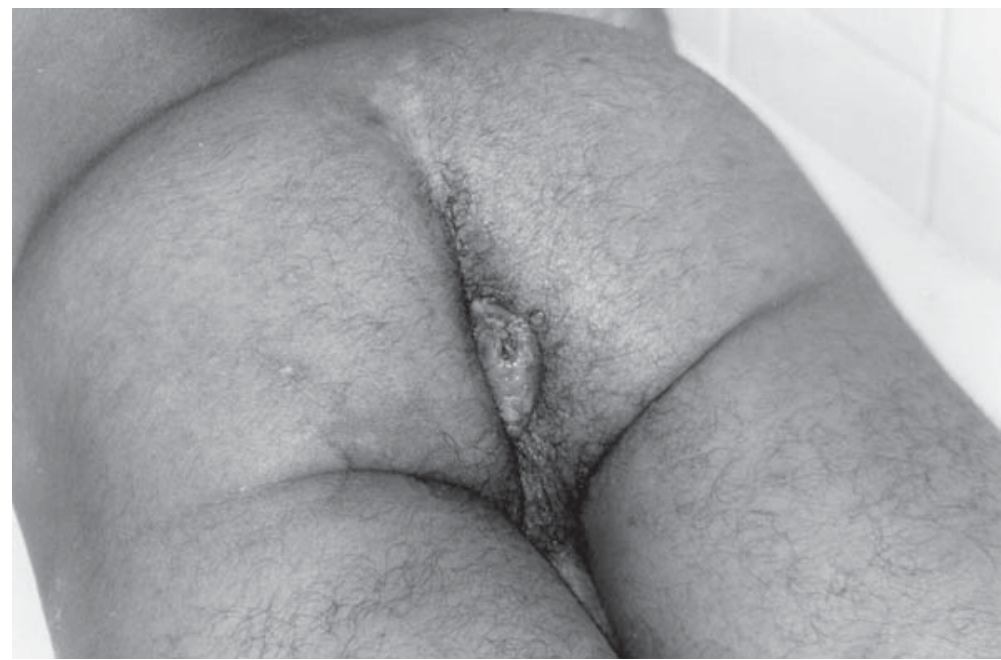

Figura 2: Miíase anal de origem não identificada.

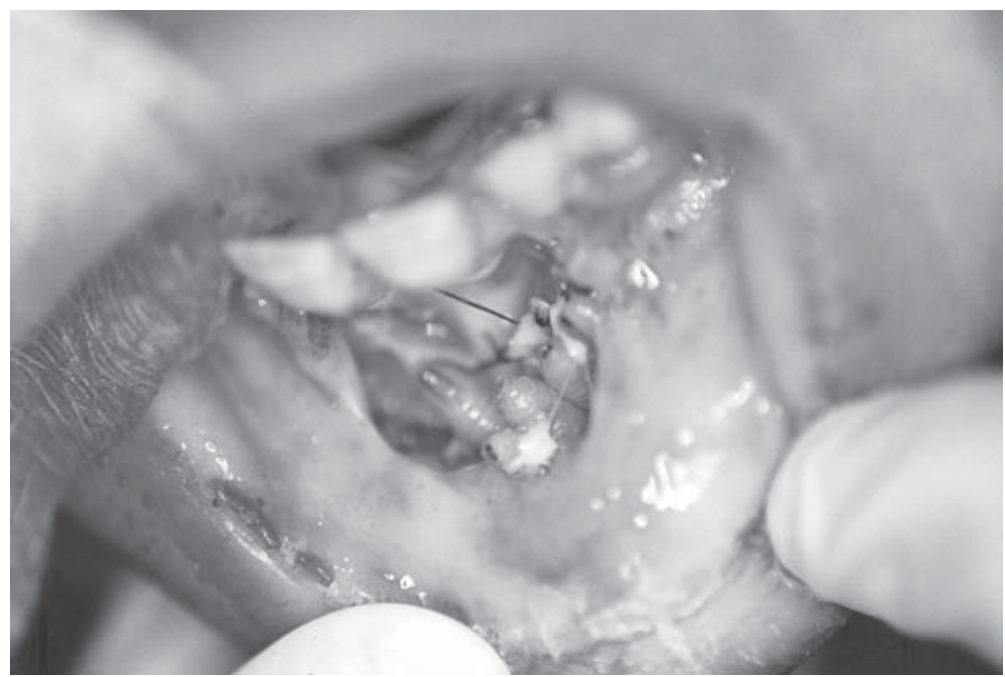

Figura 3: Miíase oral (gengiva e lábio inferior) de origem não identificada. 


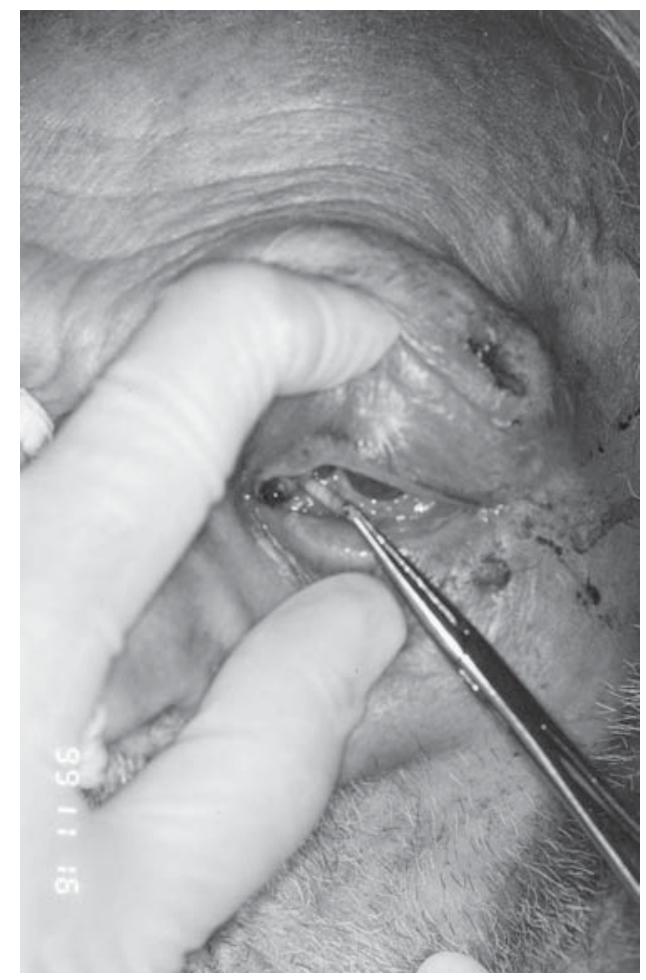

Figura 4: Miíase ocular (canal lacrimal) de origem não identificada.

morador de rua que era mendigo e apresentava-se em precário estado de higiene. Apesar disto, observamos que, em vários casos, mesmo sendo realizada a higiene corporal, as lesões estavam expostas sem nenhuma proteção, apresentando exsudação e/ou exalando odor fétido, o que acreditamos possa ser atribuído, em grande parte, ao desconhecimento do comportamento de atratividade que as moscas têm por ferimentos.

As miíases ocorreram em paciente de ambos os sexos (Tab. I) e a faixa etária variou de 08 a 93 anos (média de 55,5 anos) (Tabs. I e IV). Embora os dípteros não estejam condicionados à preferência por sexo, idade ou etnia, registra-se que as míases predominam em adultos do sexo masculino, com idade variando entre 30 a 70 anos (Durighetto Jr. et al., 1995; Kumarasinghe et al., 2000; Sherman, 2000). 


\section{Tabela III}

Sítios anatômicos de ocorrência das miíases diagnosticadas em pacientes humanos atendidos em três hospitais da rede pública na cidade do Recife-PE, no período de novembro de 1999 a outubro de 2002.

\begin{tabular}{lcc}
\hline \multicolumn{1}{c}{ Sítios Anatômicos } & Número de Casos & $\%$ \\
\hline $\begin{array}{l}\text { Membros inferiores } \\
\text { (perna, pé, tornozelo, dedo) }\end{array}$ & 10 & $41,7 \%$ \\
Cabeça & 04 & $16,7 \%$ \\
Cavidade oral & 03 & $12,5 \%$ \\
Ânus & 02 & $8,3 \%$ \\
Orelha & 02 & $8,3 \%$ \\
Olho & 02 & $8,3 \%$ \\
Região dorsal & 01 & $4,2 \%$ \\
Total & 24 & $100 \%$ \\
\hline
\end{tabular}

Diferentemente da literatura consultada, discordamos que as miíases humanas sejam de ocorrência rara. No entanto, acreditamos que os relatos desta patologia são subestimados e minimizados e que os resultados apresentados neste estudo não refletem a real situação das miíases humanas em Pernambuco, se levarmos em consideração o reduzido número de hospitais trabalhados e o fato que nem todos os casos diagnosticados nos foram informados. Além disso, acreditamos também que a maioria dos casos deve receber atendimento médico em postos de saúde da família (PSF), sendo encaminhados para os grandes hospitais apenas os casos de maior gravidade e aqueles onde não se realizou um diagnóstico adequado. A princípio, estas dificuldades poderiam ser contornadas se este levantamento pudesse ser baseado num estudo retrospectivo a partir da análise do prontuário médico dos pacientes atendidos nas unidades públicas de saúde, conforme realizado por Sherman (2000) nos Estados Unidos. No entanto, por não ser obrigatório o registro, a maioria dos profis- 


\section{Tabela IV \\ Histórico clínico dos 24 pacientes com diagnóstico de miíase, atendidos em três hospitais da rede pública na cidade do Recife-PE, no período de novembro de 1999 a outubro de 2002.}

\begin{tabular}{|c|c|c|c|c|c|c|}
\hline Caso № & Localização & Idade & Sexo & Hospital & Seqüela & $\begin{array}{l}\text { Origem da } \\
\text { Lesão/Fatores } \\
\text { Associados }\end{array}$ \\
\hline 01 & perna & 16 anos & $M$ & HGV & - & escoriações \\
\hline 02 & tornozelo/pé & 76 anos & $\mathrm{F}$ & HGV & - & $\begin{array}{l}\text { ruptura } \\
\text { vascular/diabetes }\end{array}$ \\
\hline 03 & perna & 71 anos & $\mathrm{F}$ & HGV & - & $\begin{array}{l}\text { ulceração } \\
\text { vascular/elefantíase }\end{array}$ \\
\hline 04 & pé & 76 anos & $\mathrm{M}$ & HGV & amputação & não identificada \\
\hline 05 & pé & 22 anos & $\mathrm{M}$ & HGV & amputação & $\begin{array}{l}\text { dermatite/debilidade } \\
\text { mental, desnutrição, } \\
\text { desidratação/ } \\
\text { morador de rua }\end{array}$ \\
\hline 06 & cabeça & 10 anos & $\mathrm{F}$ & HGV & - & escoriações/piolhos \\
\hline 07 & retal/anal & 64 anos & $\mathrm{F}$ & HGV & - & $\begin{array}{l}\text { Neoplasia / desnutrição } \\
\text { / anemia }\end{array}$ \\
\hline 08 & perna & 57 anos & $M$ & HGV & amputação & $\begin{array}{l}\text { ulceração vascular } \\
\text { periférica / alcoolismo / } \\
\text { elefantíase }\end{array}$ \\
\hline 09 & ânus & 29 anos & $\mathrm{M}$ & HGV & - & não identificada \\
\hline 10 & perna & 65 anos & $\mathrm{F}$ & HGV & - & escoriações \\
\hline 11 & pé & 60 anos & $\mathrm{M}$ & HGV & - & $\begin{array}{l}\text { Escoriações / } \\
\text { esquizofrenia }\end{array}$ \\
\hline 12 & cabeça & 28 anos & $M$ & HGV & - & abscesso \\
\hline 13 & cavidade oral & 58 anos & $\mathrm{M}$ & HGV & - & não identificada \\
\hline 14 & região dorsal & 22 anos & $\mathrm{M}$ & HGV & - & $\begin{array}{l}\text { ferida } \\
\text { cirúrgica/paraplegia/ } \\
\text { debilidade mental }\end{array}$ \\
\hline 15 & perna & 53 anos & $\mathrm{F}$ & HGV & amputação & traumatismo \\
\hline 16 & pé & 11 anos & $\mathrm{M}$ & $\mathrm{HCM}$ & - & traumatismo \\
\hline 17 & olho & 71 anos & $\mathrm{M}$ & $\mathrm{HR}$ & $\begin{array}{l}\text { perda da } \\
\text { visão }\end{array}$ & $\begin{array}{l}\text { Conjuntivite / } \\
\text { desidratação/ } \\
\text { debilidade física }\end{array}$ \\
\hline 18 & orelha & 09 anos & $\mathrm{M}$ & HGV & - & otite \\
\hline 19 & $\begin{array}{l}\text { boca /lábio } \\
\text { inferior }\end{array}$ & 80 anos & $\mathrm{F}$ & $\mathrm{HCM}$ & - & neoplasia \\
\hline 20 & $\begin{array}{l}\text { olho/canal } \\
\text { lacrimal }\end{array}$ & 93 anos & $\mathrm{M}$ & HGV & - & $\begin{array}{l}\text { não identificada/ } \\
\text { desidratação / diabetes }\end{array}$ \\
\hline 21 & orelha & 35 anos & $\mathrm{M}$ & HGV & - & escoriações \\
\hline 22 & cabeça & 08 anos & $\mathrm{F}$ & HGV & - & $\begin{array}{l}\text { escoriações/prurido/ } \\
\text { higiene inadequada }\end{array}$ \\
\hline 23 & cavidade oral & 24 anos & $\mathrm{F}$ & $\mathrm{HCM}$ & - & $\begin{array}{l}\text { não identificada / } \\
\text { tetraplegia / } \\
\text { debilidade mental }\end{array}$ \\
\hline 24 & cabeça & 14 anos & $\mathrm{F}$ & $\mathrm{HCM}$ & - & $\begin{array}{l}\text { escoriações/prurido/hi } \\
\text { giene inadequada }\end{array}$ \\
\hline
\end{tabular}

HGV - Hospital Getúlio Vargas; HCM - Hospital Casa de Misericórdia; HR - Hospital da Restauração. 
sionais de saúde não assinala, no prontuário médico dos pacientes, a presença de larvas de dípteros em ferimentos, o que deu o caráter de casualidade aos diagnósticos assinalados neste estudo. Acreditamos que a maioria dos diagnósticos de miíases não é registrada por razões culturais, sociais e médico-políticas e que a escassez de estudos sobre as miíases humanas no Brasil deve-se ao fato de que poucos hospitais consideram esta patologia como merecedora de registro. Pudemos também constatar que, em raras oportunidades, os profissionais de saúde adotam como conduta a retirada das larvas das lesões e o envio destas a laboratórios de referência para que as mesmas sejam identificadas, sendo a conduta de rotina a retirada das larvas e seu imediato descarte, procedimento acompanhado por expressões de nojo e reprovação com relação à situação dos pacientes.

Este é o primeiro relato da ocorrência de mí́ases humanas em Pernambuco com o envolvimento de $C$. hominivorax que demonstra a necessidade de conscientizar os profissionais de saúde da importância da identificação das espécies envolvidas como forma de conhecer o histórico e o potencial patogênico destas infestações visando a obtenção de resultados satisfatórios no tratamento, evitando assim os riscos de seqüelas indesejáveis que prolongam tanto o tratamento quanto a recuperação, aumentando o tempo de permanência dos pacientes nos hospitais.

\section{Referências Bibliográficas}

Apt, W.; Lopez, X.; Carvajal, C. \& Zulantay, I., 2001. Myiasis por Dermatobia hominis. Presentación de cuatro casos en un grupo familiar. Parasitol. Dia 25:126-128.

Calderon, O.; Rivera, P.; Sanchez, C. \& Solano, M., 1996. Cochiomyia hominivorax (Diptera: Calliphoridae) como agente causal de miiasis aural en un niño de Costa Rica. Parasitol. Dia 20:130-132.

Cunninghan, E.P., 1991. Summary of New world Screwworm. Response to an emergency. World Anim. Rev. (Special Issue):50-52. 
Durighetto Jr., A.F.; Machado, M.I.; Favoreto Jr., S. \& Magalhães, A.O., 1995. Miíases orais: aspectos clinico-laboratoriais de um caso humano. Rev. Odontol. Bras. Cent. 5:19-22.

Garcia, S.H.; Visciarelli, E.C.; Mena, F.; Gabbarini, M.; Perez, S.; Lucchi, L. \& Costamagna, S.R., 2002. Un caso de miiasis humana por Cochliomyia hominivorax (Coquerel, 1858) (Diptera, Calliphoridae) en Bahía Blanca, Argentina. Entomol. Vect. 9:591-597.

Graham, O.M., 1985. Symposium on eradication of the screwworm from the United States and Mexico. Maryland, Entomological Society of America, $68 \mathrm{p}$.

Guimarães, J.H. \& Papavero, N., 1999. Myiasis in man and animals in the Neotropical Region: Bibliographic database. FAPESP, São Paulo, $308 \mathrm{p}$.

Hall, M. \& Wall, R., 1995. Myiasis of humans and domestic animals. Adv. Parasitol. 35:257-334.

James, M.T., 1947. The flies that cause myiasis in man. U.S. Depart. Agricul. 631:1-175.

Kumarasinghe, S.P.W.; Karunaweera, N.D. \& Ihalamulla, R. L., 2000. A study of cutaneous myiasis in Sri Lanka. Int. J. Dermatol. 39:689-694.

Lobo, A.P.; Paes, M.J. \& Oliveira, J.B., 2001. Ocorrência de Dípteros Ciclorrafos na cidade do Recife. J. Bras. Patol. 37:190.

Lukin, L.G., 1989. Human cutaneous myiasis in Brisbane: a prospective study. Med. J. Aust. 150:237-40.

Maciel, M.V.; Paes, M.J.; Silva, J.S. \& Sales Jr., P.A., 2001. A Flutuação populacional de Dípteros Caliptratos no campus da Escola Superior de Agricultura de Mossoró, Esam. J. Bras. Patol. 37:50. 
Moya-Borja, G.E., 2003. Erradicação ou manejo integrado das míases neotropicais das Américas?. Pesq. Vet. Bras. 23:131-138.

Nogueira, A.S.; Leles, J.R.L.; Araújo, L.M.A. \& Lemes, R.S., 2000. Miíase humana associada a carcinoma epidermóide de lábio inferior. J. Bras. Med. 79:66-70.

Schreiber, M.; Schuckmell, N. \& Sampsel, J., 1964. Human myiasis. J. Am. Med. Assoc. 188:128-129.

Sherman, R.A., 2000. Wound myiasis in urban and suburban United States. Archiv. Int. Med. 160:2004-2014.

Stephan, A. \& Fuentefria, N.B., 1999. Miíase Oral: Parasita versus hospedeiro. Rev. Assoc. Pelot. Cirurg. Dent. 53:47-49. 